\title{
Conditioned suppression as a function of the number of stimuli that precede shock
}

JAMES A. BRAHLEK, Department of Psychology, Southern Illinois University, Carbondale, Illinois 62901

The conditioned suppression of a previously trained lever pressing response was observed in hooded rats. One group of rats received pairings of one 60-sec stimulus followed by shock. The remaining two groups received two consecutive 30 -sec stimuli with the second stimulus followed by shock. It was found that the one-stimulus group suppressed lever pressing strongly during every presentation of the stimulus. Both of the two-stimuli groups suppressed lever pressing in both stimuli on early trials. On later trials, both groups showed less suppression of lever pressing in the first stimulus than in the second stimulus, indicating the development of a discrimination of the time of shock delivery.

When pairings of a stimulus, followed by brief electric shock, are presented several times to a rat emitting operant responses, the operant response rate decreases during the stimulus and returns to the pre-stimulus rate after the shock is presented and the stimulus is withdrawn. The decrease in operant response rate during the stimulus is a demonstration of conditioned suppression.

A large number of experiments (e.g., Brady, 1955; Annau \& Kamin, 1961) have studied the conditioned suppression of operant behavior in a single stimulus that preceded shock. In the present experiment, which is similar to an experiment reported briefly by Kamin (1965), the suppression of operant behavior was observed when two stimuli preceded shock. A control group of rats received delayed conditioning trials composed of a single 60-sec stimulus that ended with shock. Two experimental groups of rats received trials composed of two 30-sec stimuli in succession, and the second stimulus ended with shock. One of the experimental groups received the trials according to a trace conditioning procedure, and the other group received the trials according to a compound (successive cue) conditioning procedure. The two experimental groups provided a comparison of response suppression in two pre-shock stimuli when the second stimulus and the intertrial stimulus were either the same (trace procedure) or different (successive cue procedure).

Subjects

METHOD

The Ss were 15 male hooded rats, 135 days old at the beginning of the experiment. Before training the lever pressing response, Ss were allowed free access to Purina Lab Chow pellets and water. Each S was tested at $80 \%$ of its ad lib weight, which was maintained during the experiment by feeding an appropriate amount of Purina Lab Chow after the daily experimental session. Apparatus

The Ss were tested during daily 60 -min sessions in a standard Lehigh Valley Electronics (LVE) operant conditioning chamber (Model $1417 \mathrm{c}$ ). Reinforcement for lever pressing was a $45 \mathrm{mg}$ Noyes rat pellet. A $1.0 \mathrm{~mA}$ shock was presented through the grid floor of the chamber by a LVE shock supply (Model 1531) and a scrambler (Model 1311SS). A light stimulus was provided by the illumination of two 7-W lamps mounted in a wall-of the chamber, and a tone $(2000 \mathrm{cps}, 80 \mathrm{~dB})$ was delivered to the chamber by a Hewlett-Packard oscillator (Model 200CD). All experimental events were automatically programmed with relays and timers, and responses were recorded on electric counters.

Procedure

On Days 1-39 of the experiment, Ss were trained to lever press on a variable-interval (VI) $1 \mathrm{~min}$ schedule of food reinforcement. On Day 40, the Ss were randomly assigned to three groups of five Ss each for the start of conditioned suppression trials. Each $S$ received six trials per day superimposed on the VI $1 \mathrm{~min}$ reinforcement schedule for the next 72 days. The intertrial interval varied from 5 to $20 \mathrm{~min}$ with a mean of $10 \mathrm{~min}$. All three groups received a 60 -sec signal on each trial before the $.2 \mathrm{sec}$ grid shock was presented, and the stimulus conditions during intertrial intervals were darkness and silence. The signal for the Light-Tone group was a $30-\mathrm{sec}$ light followed by a $30-\mathrm{sec}$ tone (successive cue procedure); for the Light-Dark group, $30 \mathrm{sec}$ of light followed by $30 \mathrm{sec}$ of darkness (trace conditioning procedure); and for the Light-Light group, $60 \mathrm{sec}$ of light (delayed conditioning procedure).

The suppression of lever pressing was measured using the suppression ratio (SR), an index that compares the rate of lever pressing during a stimulus to a reference rate of lever pressing:

$$
\text { SR }=\frac{\text { Stimulus Rate }}{\text { Reference Rate }+ \text { Stimulus Rate }} .
$$

The reference rate in this experiment was the rate of lever pressing recorded in the $60-\mathrm{sec}$ period before the light was switched on to start the trial. Two mean SRs, one for each stimulus, were computed in blocks of 18 suppression trials. The mean 18-trial SRs of the Light-Light Ss were computed for consecutive 30-sec halves (Light-1 and Light-2) of the 60-sec stimulus.

\section{RESULTS}

The first- and second-stimulus SRs of the three groups are shown in Fig. 1. The data of one Light-Light $S$ were omitted because of an apparatus failure late in suppression training. A three-factor (groups, stimuli, and trial blocks) analysis of variance on the $S R$ s yielded a significant three-way interaction $(F=2.80$, $\mathrm{df}=46 / 253, p<.01)$, which permitted a simple main effect analysis of each of the factors (Winer, 1962, p. 323). The simple main effects reported are significant at the .05 level.

Differences in first-stimulus SRs were found on trial Blocks 19-24. At each of these points, the Light-Light group suppressed responding strongly in Light-1, whereas the Light-Tone and Light-Dark groups suppressed substantially less. The latter two groups showed the same decreasing level of first-stimulus suppression across trials.

The second-stimulus suppression of the Light-Light group was nearly complete across blocks and was uniformly greater than the second stimulus suppression of the Light-Dark group. The secondstimulus suppression of the Light-Tone group was the same as that of the Light-Dark group on Blocks 1-7 (except at Block 2). By Block 10, the second-stimulus suppression of the Light-Tone group increased to the level of the Light-Light group.

The difference in suppression between stimuli was also tested for each group. During Blocks 1-6, the Light-Tone group suppressed more in the first stimulus than in the second stimulus. The direction of this difference was reversed during Blocks 9-24. Similarly, the Light-Dark group suppressed more in the first stimulus during Blocks $1-4$ and more in the second stimulus during Blocks 12-24. There was no significant difference between Light-1 and Light-2 suppression for the Light-Light group.

$$
\text { DISCUSSION }
$$

The present data show that the conditioned suppression of lever pressing is related to the number of stimuli that precede shock. When one stimulus preceded shock, the suppression of lever pressing was nearly complete during both halves of the stimulus. When two consecutive stimuli preceded shock, however, the initially strong suppression in the first stimulus became weaker as training continued, and the initially moderate suppression in the second stimulus either remained moderate or became stronger as training continued.

One difference between the two-stimuli and one-stimulus procedures may be the adequacy of each procedure in providing cues that are differentially related to the time of shock delivery. (See Sheffield, 1965, for a similar discussion related to salivary conditioning in the dog.) When two stimuli precede shock, the second stimulus is followed immediately by shock, but the first 


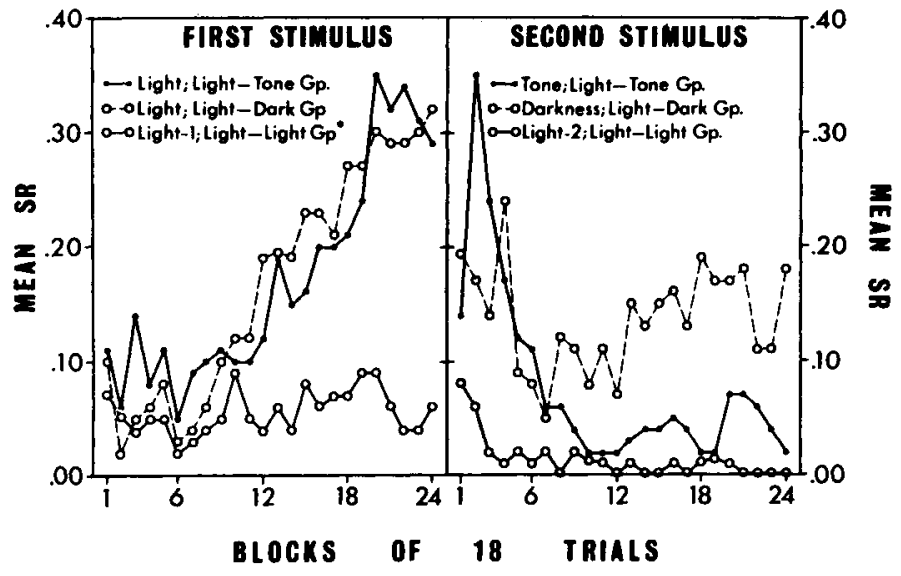

Fig. 1. Mean first- and second-stimulus suppression ratios as a function of trial blocks for Light-Tone, Light-Dark, and Light-Light groups.

stimulus is followed by the same shock after a delay equal to the duration of the second stimulus. This delay of shock may account for the weaker suppression of lever pressing that developed during the light for the Light-Tone and Light-Dark groups. The one-stimulus procedure does not provide cues that are differentially related to shock, with the result that strong suppression of lever pressing is maintained throughout the stimulus.

The present data also suggest that the level of suppression during the second of two consecutive stimuli is related to the degree of similarity between the second stimulus and the intertrial stimulus. When the second stimulus and the intertrial stimulus were different (Light-Tone group), second-stimulus suppression became strong, but when the two stimuli were the same (Light-Dark group), second-stimulus suppression was weaker. The reason for this effect appears related to the development of a second discrimination, one between the intertrial interval and the pre-shock warning signal. For example, the trace conditioned Ss apparently learned that darkness may be either a safe stimulus or a fear stimulus, depending on preceding cue conditions. Darkness preceded by shock was shock-free, but darkness preceded by the trace CS (light) was associated with an impending shock. The fact that some responding did occur following the trace CS indicates that a trace procedure taxes the rat's ability to discriminate past cues. The successive cue procedure, however, made the discrimination one of present rather than past cues by superimposing a tone on the darkness prior to shock. In effect, Ss trained with the successive cue procedure had only to discriminate darkness (shock-free signal) from the combination of tone and darkness (shock signal) and to respond accordingly.

\section{REFERENCES}

ANNAU, Z., \& KAMIN, L. J. The conditioned emotional response as a function of intensity of the US. Journal of Comparative \& Physiological Psychology, 1961, 54, 428-432.

BRADY, J. V. Extinction of a conditioned "fear" response as a function of reinforcement schedules for competing behavior. Journal of Psychology, $1955,40,25 \cdot 34$.

KAMIN, L. J. Temporal and intensity characteristics of the conditioned stimulus. In W. F. Prokasy (Ed.), Classical conditioning: a symposium. New York: Appleton-Century, 1965. Pp. 118-147.

SHEFFIELD, F. D. Relation between classical conditioning and instrumental learning. In W. F. Prokasy (Ed.), Classical conditioning: a symposium. New York: Appleton-Century, 1965. Pp. 302-322.

WINER, B. J. Statistical principles in experimental design. New York: McGraw-Hill, 1962.

\section{NOTE}

1. This report is based on a thesis submitted to Southern Illinois University in partial fulfillment of the requirements for the MA degree. The author wishes to thank Donald Meltzer and Robert M. Herrick for their assistance. 